\title{
Presenting a New Wireless Strain Method for Structural Monitoring: Experimental Validation
}

\author{
Amedeo Gregori, ${ }^{1}$ Emidio Di Giampaolo, ${ }^{2}$ Alessandro Di Carlofelice, ${ }^{2}$ and Chiara Castoro ${ }^{1}{ }^{1}$ \\ ${ }^{1}$ Department of Civil, Building and Environmental Engineering, University of L'Aquila, Via Giovanni Gronchi 18, \\ 67100 L'Aquila, Italy \\ ${ }^{2}$ Department of Industrial and Information Engineering and Economics, University of L'Aquila, Via Giovanni Gronchi 18, \\ 67100 L'Aquila, Italy \\ Correspondence should be addressed to Chiara Castoro; chiara.castoro@libero.it
}

Received 14 March 2019; Revised 7 July 2019; Accepted 23 August 2019; Published 3 November 2019

Guest Editor: Ye Lu

Copyright (c) 2019 Amedeo Gregori et al. This is an open access article distributed under the Creative Commons Attribution License, which permits unrestricted use, distribution, and reproduction in any medium, provided the original work is properly cited.

\begin{abstract}
The structural health monitoring (SHM) of large and complex infrastructures as well as laboratory tests of new structures and materials resorts to strain gauge measurements to check mechanical stress. A wireless measurement of the strain gauge response is desirable in many practical applications to avoid the cost and the difficulty of wiring, particularly in large structures requiring several sensors and in complex objects where the measurement points are difficult to access. In this paper, a wireless strain gauge which is a hybrid between an RFID tag and a usual thin-film resistive strain gauge is experimented. Installation and maintenance problems of the wireless sensor networks are overcome allowing a high level of measurement accuracy, comparable to that of wired strain sensors, together with a long measurement distance. A large set of measurements has been performed using reference specimens and readings in order to validate the sensor and to develop a calibration procedure that makes the sensor suitable for a large number of different applications in civil engineering.
\end{abstract}

\section{Introduction}

The structural monitoring of large and complex infrastructures requires the use of a number of specific sensors distributed on a large area or volume to form a monitoring system composed of independent or interconnected sensor nodes like a smart skin. Because of the complexity of that monitoring system, some kind of intelligence is required to make easy the managing of the system and the handling of the large amount of measured data. In particular, the communication between the sensor nodes and a managing unit that collects and stores the information gathered from the sensors spread over the structure is a critical issue that preferably has a solution with wireless systems. In fact, wireless sensors permit a fast and easy installation even in points difficult to access while their cost is lower than that of a wired system.
Wireless sensor networks (WSNs) are a favorite candidate for structural health monitoring (SHM) of large structures. As an interdiscipline consisting of sensor, communication, and wireless technology, WSNs were initially applied in the military and then extended to environmental monitoring, agriculture, medical treatment, and civil engineering [1-13]. Various types of sensors have been developed during the past decades (such as strain gauge and optical fiber sensors) while SHM technology is becoming important in a wide range of technical fields, most of the time in combination with structural use of special composites and high-performance materials [14]. Actually, it is more and more understood as the SHM system can improve safety and reliability of structures by autonomously monitoring the conditions or detecting critical damage. In [9], authors state that in comparison with traditional wired sensor networks, wireless systems for SHM have numerous advantages in terms of better 
flexibility, software or hardware expandability, cost effectiveness, and fault tolerance. Interesting applications of wireless sensors made in aerospace, civil, and environmental engineering are discussed in [15-18] comparing the practical needs concerning space requirements and cost increments with those of traditional wired techniques. In [19], a dipole antenna-based wireless sensor for the damage detection of a composite rotor blade is investigated. In [20], the use of WSNs has been presented as a useful tool even in forest fire surveillance, allowing real-time acquisition, evaluation, and analysis of environmental information including temperature, humidity, sound, vibrations, and smoke as well as pictures of buildings and forest. Some advances in research, development, and implementation of smart sensor networks and health monitoring systems for civil infrastructures are presented in $[21,22]$. In particular, cases of study of WSNs and their integrated systems and implementations in offshore platform structures, hydraulic engineering structures, large span bridges, and large space structures have been reported. Other researches on wireless sensing technology applied to SHM for buildings and civil engineering structures are discussed in [23].

During the entire last decade, implementation of wireless transmitters for continued structural damage monitoring became a promising research field to be often related to new patented inventions and devices. In [24], the authors present a new methodology for operating a monitoring system that provides near-real-time structural condition assessment for extreme events and long-term deterioration information, using MEMS-type accelerometers. This proposed structural monitoring system comprises modular, battery-powered data acquisition devices which transmit structural information to a central data collection and analysis device over a wireless data link. Data acquisition devices comprise mechanical vibration sensors, data acquisition circuitry, wireless transmitter, and battery. For sophisticated analysis after a natural hazard or extreme event, the authors suggested that powerful computers may be interfaced with the central device.

While WSNs have been extensively investigated in recent years, many practical challenges are still to be faced when employing such a technology for many SHM applications, including civil and mechanical infrastructures [25]. In fact, a WSN must remain in operation over multiple decades with maintenance costs low enough to justify its integration into a given structural maintenance strategy. These technical barriers include ensuring reliable power sources for sensor nodes, reducing installation and maintenance costs, and automating the collection and analysis of data acquired by a WSN.

As a possible solution to overcome the mentioned challenges, in [26] is discussed the use of sensor nodes that collect measurements from a structure in a completely passive manner without any electrical power. In [27], a wireless sensor network with temperature-compensated measuring technology for long-term structural health monitoring of buildings and infrastructures is presented. A brief summary and comparison among benefits and disadvantages related to active and passive wireless sensors are given in [28] together with the presentation of a passive wireless structural health monitoring sensor made with a flexible planar dipole antenna. In fact, it is understood that chipless passive wireless sensors can give real-time structural information for SHM without space and battery constraints in harsh environmental conditions [10-13]. Chipless passive wireless strain and damage detection sensors based on a frequency selective surface are presented in [29].

In this paper, a new kind of sensor node for mechanical stress detection which is obtained as a hybridization of an RFID (Radio Frequency Identification) tag and a resistive strain gauge is exploited.

It is a semipassive wireless strain sensor tag, which uses a piezoresistive thin-film strain gauge (like a wired sensor), but it can be passively interrogated as an RFID tag. Like an RFID system, there is an interrogation unit (i.e., a commercial RFID reader) that radiates an electromagnetic wave that impinges on the antenna of the sensor tag waking it up. The strain gauge varies its resistance in accordance with the applied strain and drives an oscillating circuit that modulates the electromagnetic wave backscattered by the sensor tag antenna. The modulating frequency is dependent on the applied strain and can be easily measured by means of a spectrum analyzer or a frequency meter once the modulated backscattered wave is received back by the interrogation unit.

The prototype of the proposed sensor tag makes use of a battery to power the oscillating circuit that is maintained turned off for all the time except for a short time interval during the measurement interrogation. For this reason, the life span of the battery can be very long. A detailed description of the sensor tag is reported in [30] and in Materials and Methods.

Since each sensor tag is autonomous and independent from other nearby deployed sensors, the resulting network has the simple star topology where the central unit is an RFID reader that interrogates one-by-one all the deployed sensor tags. In this paper, the application of this sensor tag to realistic cases concerning the field of civil and mechanical engineering is shown. The assessment of the sensor tag is demonstrated by means of measurements of the Young's modulus of different materials and by a comparison with the results obtained using a calibrated wired system. A calibration procedure of the new sensor tag has been developed, and a detailed measurement campaign using many instrumented specimens is also reported.

With this study, we have assessed the possibility to transfer the measured information from the sensor tag to a central unit analogically avoiding the analog to digital conversion at the sensor tag level but performing it at the level of the central unit. We transmit the measured data analogically as a backscattered frequency modulated continuous signal that can be sampled at the receiving unit using an appropriate device having a high data rate so that very fast phenomena can be detected easily, with high accuracy, using cheap sensor tags. The feasibility of this new sensor tag has been investigated; a measurement campaign shows its effectiveness and proves the advantages of this new sensor tag in particular in measuring vibrations and dynamic phenomena. The managing of the system results is simple because it does not suffer of 
restriction on the data rate, since the strain information travels over analogical signals.

\section{Materials and Methods}

The overall SHM system consists of an interrogating unit (i.e., a reader) placed in a convenient position (e.g., near the ground in Figure 1) and several sensor tags deployed on the structure to be monitored (e.g., in positions difficult to access). Tags are fixed to the structure; they are designed to remain operative for several years, while the interrogation unit is intended to be portable and placed at the measurement location only when needed (obviously, it can be also used for a permanent monitoring of the structure). All sensor tags are quiescent (i.e., inactive) for all the time except for the short time interval when interrogated. During interrogation, they measure the strain affecting the portion of the structure where each of them is stuck on and send back to the interrogation unit a signal encoding the strain measured value. The optimal operation of the system is achieved when the interrogation unit has a line of sight with each sensor tag, but the system is able to work even in nonline of sight conditions. As sketched in Figure 1, the distance between the interrogation unit and the sensor tags can be of several meters. Like in logistic applications, the number of tags that can be handled by a single interrogation unit is large (e.g., a commercial reader can interrogate several tags per second), but that number can be larger or smaller in agreement with the repetition time of interrogations. In case of static objects (i.e., the change of the status of the monitored structure is slow compared to the measurement time), the interrogation rate can be low (the number of tags interrogated in the time unit is not an issue), and as a consequence, the number of tags that can be handled by an interrogation unit is limited only by the maximum interrogation distance, i.e., the distance over which the tag is not getting enough power to be woken up.

The developed sensor consists of three main circuital blocks: an RFID block, a supply block, and a sensing block as shown in Figure 2(a).

The RFID block consists of an antenna, a commercial RFID microchip (i.e., an NXP GMiL+), and a pin diode with its feeding network. The antenna is a dipole-like antenna operating at $868 \mathrm{MHz}$; the pin diode is connected to the antenna terminals by means of a feeding network, and it is used to modulate (on-off) the backscattered signal. The RFID microchip, powered by an external battery, allows the reading/writing distance of the sensor tag up to $30 \mathrm{~m}$ (nominal) and allows the remote control of the voltage level of a logic pin by means of an appropriate writing of the configuration word in its memory. This voltage level is used to switch the supply and sensing blocks on and off.

The sensing block is essentially a resistance-to-frequency converter circuit whose output is a squared wave signal that is used to drive the pin diode connected to the antenna terminals. Under the squared wave signal, the input impedance of the pin diode switches between two values (low and high impedances) performing an amplitude modulation of the electromagnetic wave that is backscattered by the antenna (Figure 2(a)). Since the frequency of the squared wave signal

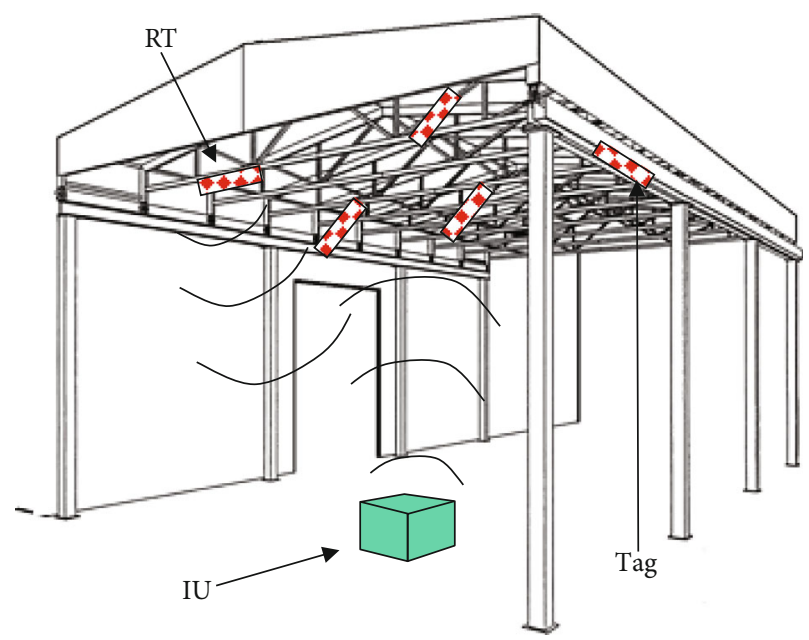

FIGURE 1: Scheme of the system measurement set-up. Tags are represented as dotted rectangles; the interrogation unit (IU) is shown as a box on the ground while RT means responding tag.

is proportional to the strain gauge stretch, the backscattered signal, amplitude modulated by the squared wave signal, carries the information concerning the strain measured by the strain gauges.

Details of the resistance-to-frequency converter are shown in Figure 2(b). It is composed of a full Wheatstone bridge strain gauge circuit (i.e., four piezoresistive thin-film strain gauges like in a wired sensor) and an operational amplifier that amplifies the small voltage changes across the bridge.

Actually, the Wheatstone bridge is a well-known circuit consisting of four resistive arms with resistances $R_{1}, R_{2}, R_{3}$, and $R_{4}$ and an excitation voltage, $V_{\mathrm{e}}$, applied across the bridge. The output voltage $V_{\mathrm{o}}$ is zero when $R_{1} / R_{2}=R_{4} / R_{3}$ and the bridge is said to be balanced. Any change in resistance in any arm of the bridge will result in a nonzero output voltage.

A possible configuration for strain measurements is the so-called quarter-bridge. It has a strain gauge at one arm of the bridge (active arm), e.g., $R_{4}$, which is the only changing resistance of the bridge. $R_{4}=R_{0}+\Delta R$, where $R_{0}$ is the unstressed resistance (or nominal resistance) of the strain gauge and $\Delta \mathrm{R}$ models the strain-induced change in resistance due to an applied strain $\varepsilon$.

It is $\Delta R=R_{0} \cdot \mathrm{GF} \cdot \varepsilon$, where GF is the Gauge Factor, i.e., the sensitivity of the strain gauge to strain which for metallic strain gauges is typically around 2 .

If we assume that $R_{1}=R_{2}$ and $R_{3}=R_{0}$, the output voltage is

$$
V_{\mathrm{o}}=-\frac{\mathrm{GF} \cdot \varepsilon}{4}\left[\frac{1}{1+\mathrm{GF} \cdot \varepsilon / 2}\right] V_{\mathrm{e}}
$$

In practice, considering that the strain measurements rarely involve quantities larger than a few millistrain, the output voltage (Equation (1)) cannot be larger than a few thousandths of the excitation voltage. For these reasons, wired 


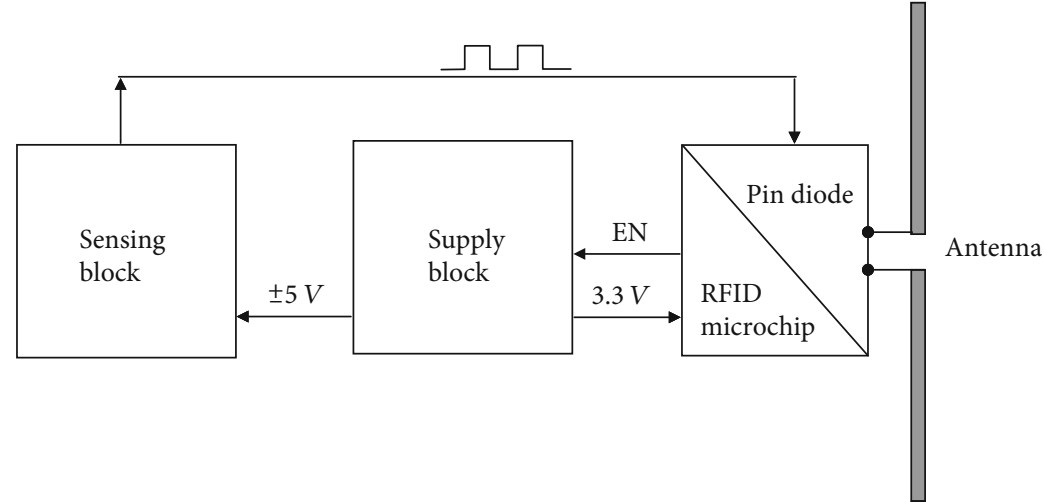

(a)

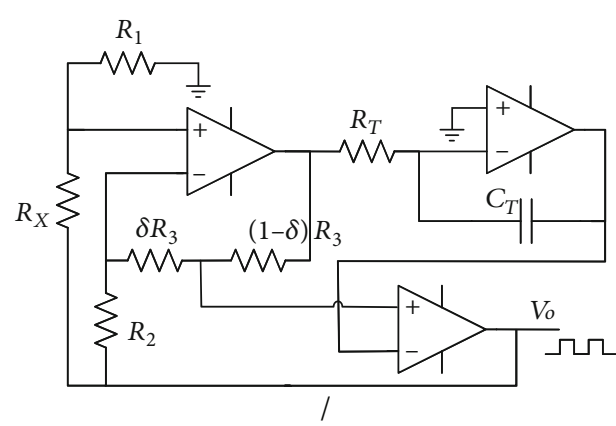

(b)

FIGURE 2: (a) Scheme of the sensor tag; three main circuital blocks: an RFID block, a supply block, and a sensing block. The sensor tag is quiescent and can be activated on demand. (b) Schematic of the resistance-to-frequency converter circuit.

systems resort to an amplification of the output voltage to bring the signal to a level where it can be conveniently handled for indication or recording. In the proposed wireless system, instead, the resistance-to-frequency technique is used in order to codify the strain measurement into a modulation frequency while preserving the high sensitivity of the Wheatstone bridge. A deeper description of the resistanceto-frequency converter is provided in [30].

The unbalance voltage due to the resistance change is then integrated, and its polarity is fed back to the bridge as the bias voltage to sustain the oscillation. The oscillation frequency changes quite linearly with the stretching of the strain gauges. The output of this circuit drives a pin diode circuit for modulating the backscattered signal.

The interrogating unit consists of a commercial RFID reader (the same kind used in logistics) and a spectrum analyzer connected to an antenna and to a personal computer (Figure 3); alternatively, we have used a Universal Software Radio Peripheral (National Instruments NI USRP 2920) particularly suited for dynamic measurements. At the start of measurements, the interrogation unit sends, by means of the RFID reader, an electromagnetic wave which delivers both the energy to wake up the tags and a query command to boost the tags to reveal themselves. Awaken tags modulate the electromagnetic wave that scatters back from their antennas with a random numeric code revealing their readiness for communication. Then, the RFID reader performs the inventory of all the responding tags which identify themselves sending back their ID. Once the inventory is completed, the interrogation unit starts with the measurements of the strain gauge status of the inventoried tags.

The measurement procedure is repeated identically for each one of the responding tags and consists of three steps. In the first step, the interrogation unit opens a specific reading/writing session with the $i$ th responding tag identified by means of its ID and enables that tag (with appropriate writing of the configuration word located in the memory of the tag) to switch on the supply circuit block which supplies energy to the sensing block. After that, during the second step, the interrogation unit sends a continuous wave (CW) signal

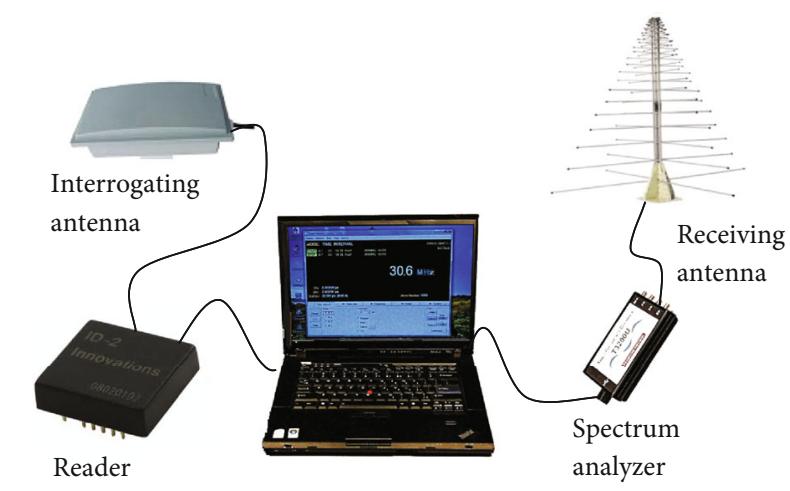

FIGURE 3: The interrogation unit consists of a commercial RFID reader and a spectrum analyzer connected to an antenna and to a personal computer.

and waits for the signal backscattered from the tag which delivers the strain gauge status information. In fact, the strain gauge varies its resistance in accordance with the applied strain and drives an oscillating circuit (inside the sensing block) which modulates the electromagnetic wave backscattered by the tag antenna. The modulating frequency is linearly dependent on the applied strain, so the backscattered signal has a different frequency modulation in accordance with the strain gauge status. Once the modulated backscattered signal is received by the interrogation unit, the modulating frequency is easily measured by means of a spectrum analyzer and the strain status is retrieved. Once the strain measurement is completed, the interrogation unit becomes the step three. A new reading/writing session is open to disable (by writing the appropriate memory location of the tag) the supply circuit block, and consequently, the sensing block is switched off. The modulation of the backscattered signal finishes, and a CW signal arrives to the interrogating unit which closes the communications with that tag and starts to interrogate another inventoried tag repeating the above-described three steps. Therefore, the sensing circuit is kept turned off all the time except for the short time interval during the measurement interrogation (step two). 


\section{Set-Up of the Experimental Tests}

To evaluate the effectiveness of the proposed sensor tag, a campaign of measurements has been performed. Tasks of this experimental activity were the calibration of the sensor and the comparison of the wireless measured data with those achieved with a consolidate method based on wired sensors. Specific tests have been also performed to estimate the maximum interrogation distance for the wireless sensor. Inside a laboratory room, with several obstacles making this environment noisy, the sensor tag was proved to allow for an interrogation distance even larger than 20 meters.

To prove the effectiveness of the proposed new wireless strain measurement technique, the elastic properties of three materials have been investigated using wireless sensor tags in place of the wired strain gauges. In particular, the experimental procedure described in this section refers to the elastic modulus assessment of brass, aluminium, and steel samples, respectively. These estimations have been first performed in accordance with a common wired strain gauge technology. Subsequently, the estimation of the elastic modulus of the three materials has been repeated on the base of the wireless strain measurements. The two procedures are discussed in Results and Discussion, together with a comparison of the experimental results.

The experimental tests were performed in accordance with the wired strain measurement technique, and those concerning the new proposed method have been all carried out operating a specific apparatus. In particular, the experimental apparatus included a manual compensator (working with the principle of the Wheatstone bridge) and a vertical metal frame (Figure 4) that mainly consists of a rigid steel-made structure built for a laboratory test and standing in the vertical plane and designed to apply controlled forces up to $10 \mathrm{kN}$ in tension. In particular, a manually driven wheel is mounted on the top of the testing frame. The wheel controls a lever mechanism by which a variable tensile force is introduced in the specimen causing it to extend. A load cell mounted in series with the specimens (and electrically connected to the compensator) allows the measurement of the tensile force applied during the tensile test.

Operating the just mentioned apparatus in a proper way, the elastic modulus $E$ (Young's modulus), the shear modulus $G$, and/or the Poisson's ratio $v$ of any elastic material can be assessed. So far, only specimens of material previously instrumented with wired strain gauges have been considered for tests.

Three different solid metal pieces made of brass, aluminium, and steel, respectively, have been selected and machined to obtain a couple of identical specimens from each of them. The two specimens of each couple have been assumed to be homogeneous and to univocally represent the same material. To perform tensile tests, the specimens had to be previously worked and instrumented. Once machined, specimens have been characterized from a dog's bone shape, with the two hands kept bigger to secure the specimen to the testing apparatus and to favorite a regular strain diffusion along the central, cylindrical portion of the specimen (stalk) when applying tensile forces. The cylindrical portion of the speci-

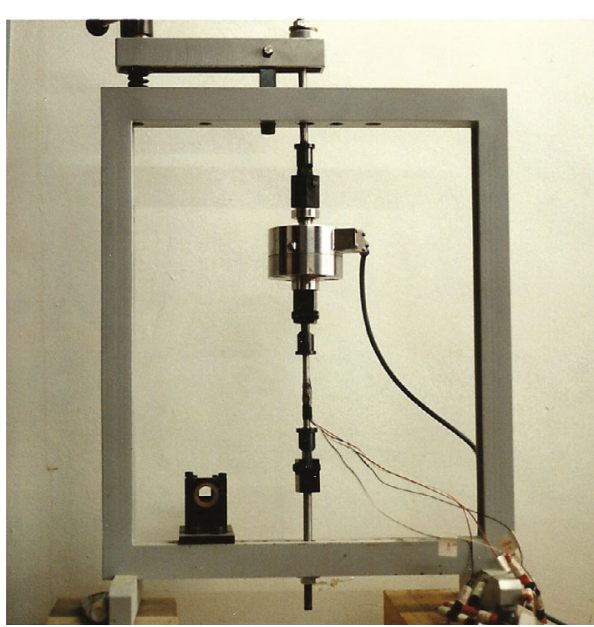

FIgURE 4: Steel frame for the laboratory tests.

mens measures $8 \mathrm{~mm}$ in diameter and about $100 \mathrm{~mm}$ in length (Figure 5).

During the test, the applied tensile forces have been measured and controlled by means of a load cell mounted in series with the specimen and electrically connected to the compensator. The load cell is characterized by a maximum load capacity of $20 \mathrm{kN}$, a maximum output signal of $4000 \mathrm{mV} / \mathrm{V}$, and a scale factor $k=2$.

The number and the magnitude of the load increments applied during the tensile test have been set in accordance with the different strengths and stiffness of the various metal specimens. In particular, load increments have been limited in the range of $0.75 \div 1 \mathrm{kN}$ (equivalent signal variation: 150 $\div 200 \mathrm{mV} / \mathrm{V}$ ) and have been repeated several times upward and downward, respectively. A complete load sequence includes a number of steps upward to a maximum load value and an equal number of load decrements downward to a zero load value, so to obtain a closed loop. The maximum applied load has been fixed in advance and kept low enough to avoid the specimens to yield: about half of the yield threshold was reached as maximum stress.

According to the elastic theory, tensile strains resulted in the specimen while loads are being applied. During the experiments, tensile strains have been detected and measured by means of several strain gauges previously mounted on the specimen and electrically connected to the compensator. In particular, each specimen has been instrumented with two single grid strain gauges glued on the opposite side of the specimen, the grids being aligned with the longitudinal axis of the specimen. Common strain gauges 3/120LY4x type, characterized from $R=120 \Omega$ and a scale factor $k=2$, were adopted.

The number of strain gauges used in each single test varies from two to four according to the half or to the whole bridge configuration, respectively. The reason for multiple gauges mounted on the same specimen first comes from the undesired possibility that applied tensile force could act eccentrically with respect to the ideal longitudinal axis of the specimen. In fact, applied loads acting with casual eccentricities introduce undesired bending actions so that a not 


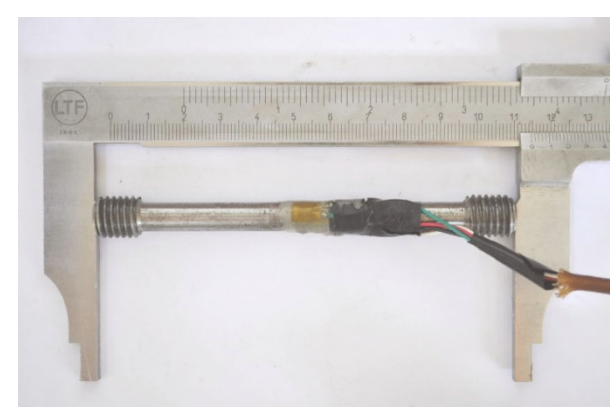

FIGURE 5: One of the instrumented steel specimens.

uniform strain distribution across the transversal section of the specimen may result. An average signal computed from strain gauges mounted on opposite sides of the same specimen allows to compensate for this undesired effect and accounts for the ideal, uniform tensile strain to be considered in the tensioned specimen. On the other hand, when more arms of the Wheatstone bridge have been made active, the sensitivity of the bridge increased. Moreover, due to possible temperature change during the test, the need for a second signal compensation rises as well. For this reason, signals from gauges mounted on the loaded specimen are combined with those recorded, at the same time, from gauges mounted on a second, identical specimen kept unloaded. In this research work, both the mechanical and thermal compensations of the strain measurements have been performed at once, recording signals from gauges in a whole bridge configuration.

\section{Results and Discussion}

The elastic modulus of the three materials has been determined by means of tensile tests performed in accordance with the wired method first, then using wireless strain gauges.

\subsection{Experimental Data from the Wired Method. Experimen-} tal curves obtained from separate tensile tests carried out on the brass specimen, on the aluminium specimen, and on the steel specimen, respectively, have been reported in the graph of Figure 6. The values of the applied stress $\sigma$ have been reported on the vertical axis and measured strains $\varepsilon$ in the horizontal one.

As expectable, the experimental data clearly proved the elastic behavior of the specimens. According to Hooke's law $(\sigma=E \cdot \varepsilon)$, the estimation of the elastic modulus $E$ of each different material was then obtained as a slope (angular coefficient) of the linear regressions built on the experimental data. Both the ascending and descending branches of each experimental curve have been considered in these calculations. In fact, it is worth to note that a curve actually consists of a very narrow loop in which the ascending and the descending branches, respectively, do not precisely overlap each other. This fact (undesired) is usually assumed to depend from hysteretic phenomena (unavoidable) taking place during the loading/unloading cycles. In our research, the loop amplitude was made sure to remain always negligible and not to affect the test repeatability. This explains

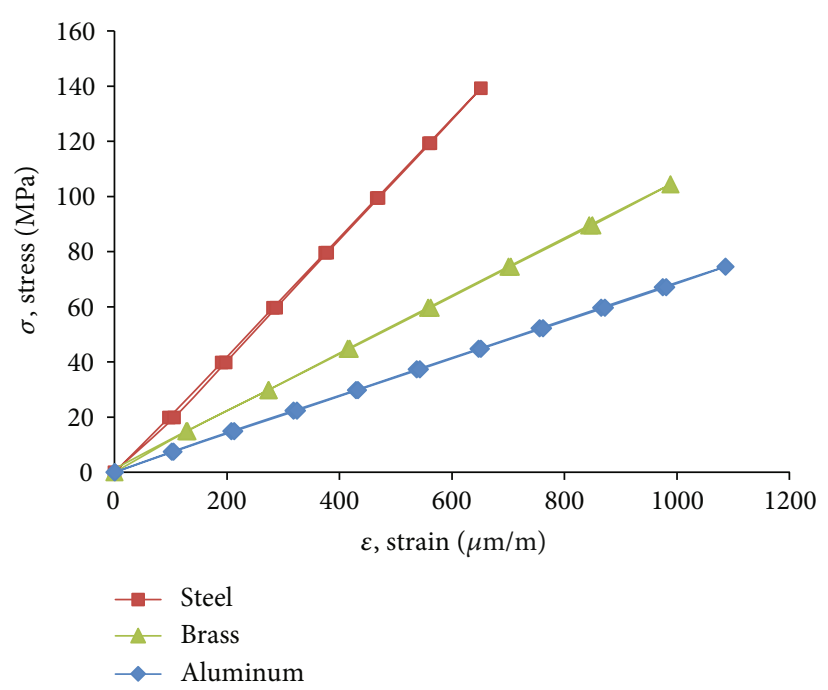

FIgURE 6: Experimental stress-strain curves obtained from tensile tests on steel, brass, and aluminium specimens using the wired method.

why loops are not clearly visible from curves plotted in Figure 6.

On the other hand, linear regressions built on experimental data are found to be very accurate and characterized by $R^{2}$ values almost equal to 1 . In this experimental work, the mean value of the three measurements repeated on each metal specimen has been assumed as the reference value for the elastic modulus $E_{\text {ref }}$ of the considered material. Single experimental results and $E_{\text {ref }}$ values have been reported in Table 1.

4.2. Experimental Data from the Wireless Method: Static Measurements. To prove that the wireless sensor tag is a reliable measuring tool, the elastic modulus assessment previously carried out on the base of the wired method has been attempted in the wireless mode. As before, tensile loads (and stresses) continued to be measured by means of the same load cell and compensator.

Data plotted in Figure 7 show the results of a tensile test performed on the brass specimen and recorded in accordance with the new wireless method. Values of the applied stress $\sigma$ have been reported on the vertical axis, and the recorded bridge frequency changes on the horizontal one. To be consistent in the experimental procedure, stress increments and load sequence have been repeated as in previous tests carried out to estimate reference elastic modulus $E_{\text {ref }}$ of the brass specimen in accordance with the wired method.

Experimental data in Figure 7 highlight that a relationship exists among the applied load increments and the frequency changes (i.e., the modulation frequency of the backscattered signal) measured from the interrogation unit. Actually, a linear regression built on the experimental data was found to be very satisfactory, with the coefficient of determination $R^{2}$ approaching unit $\left(R^{2}=0.9931\right)$. On the other hand, considering experimental data more into details, it is noted that the experimental curve yet consists of a very narrow loop (as for wired gauge strain measurements) in 
TABLE 1: Estimation of the reference elastic modulus $E_{\text {ref }}$ of the materials and $R^{2}$ of the single linear regressions. Wired method.

\begin{tabular}{|c|c|c|c|c|c|c|c|c|c|}
\hline \multirow{2}{*}{ Material } & \multirow{2}{*}{ Stress step $(\mathrm{MPa})$} & \multirow{2}{*}{ Max. stress $(\mathrm{MPa})$} & \multicolumn{2}{|c|}{ Cycle 1} & \multicolumn{2}{|c|}{ Cycle 2} & \multicolumn{2}{|c|}{ Cycle 3} & \multirow{2}{*}{$E_{\text {ref }}(\mathrm{MPa})$} \\
\hline & & & $E_{1}(\mathrm{MPa})$ & $R_{1}^{2}$ & $E_{2}(\mathrm{MPa})$ & $R_{2}^{2}$ & $E_{3}(\mathrm{MPa})$ & $R_{3}^{2}$ & \\
\hline Steel & 20 & 140 & 219200 & 0.9999 & 223700 & 0.9999 & 223100 & 1.000 & 222000 \\
\hline Brass & 15 & 105 & 106100 & 0.9995 & 106600 & 1.000 & 105400 & 0.9996 & 106000 \\
\hline Aluminium & 7.5 & 75 & 68300 & 1.0000 & 69200 & 1.000 & 68900 & 1.000 & 68800 \\
\hline
\end{tabular}

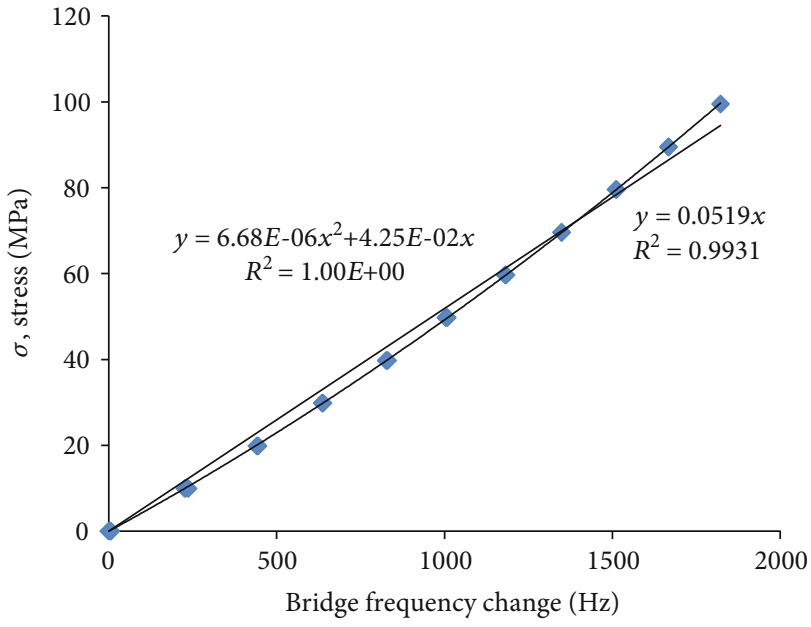

FIGURE 7: Wireless sensor tag frequency variation recorded during the tensile test on the brass specimen.

which the ascending and the descending branches tend to a unique monotonic curve perfectly fitted by a second order polynomial regression $\left(R^{2}=1.00\right)$ (Figure 7$)$. This was confirmed also for different materials.

About the weak nonlinearity of the experimental curve, it is worth to note that it mainly depends on the actual value of the hardware parameters, i.e., the tolerances of the electronic components (in particular, resistances and capacitances) that make the response of the sensor tag not perfectly linear as explained in [30]. Fortunately, the level of nonlinearity can be made small by means of an accurate choice of the electronic components so that the resulting nonlinearity can be ignored as shown by the $R^{2}$ value calculated for the linear regression plotted in Figure 7, approaching unit.

On the other hand, observing the four curves of Figure 8, referring to tensile tests carried out on a unique aluminium specimen, it can be noted that they significantly differ from each other for the entity of the frequency increments recorded in consequence of the same strain variation produced in the specimen. This is because the frequency response of the sensor tag changes in the four experiments; in particular, the frequency domain and the slope of the response are different. The change of the frequency response is achieved by means of different choices of the values of the electronic components of the oscillating circuit [30]. Therefore, the sensor tag can be tuned to have different frequency responses, and each response is characterized by a different reference frequency value $f_{0}$ (i.e., the frequency in unstressed condition). For these reasons, the four series of measure- ments shown in Figure 8 have been operated into different domains and have different slopes.

Linear regressions plotted in Figure 8 are characterized from different slopes $\Delta \sigma / \Delta f$ and clearly show the highest bridge frequency changes when using the sensor tag characterized by the lowest initial frequency $f_{0}=8648 \mathrm{~Hz}$ (red square dots curve). Consequently, the sensor tag characterized by higher initial frequencies results to be less and less sensitive to the same stress (strain) increments applied to the specimen.

4.3. Calibration. According to the wired method, a unique, direct proportion exists among the strain variations, the gauge resistance changes, and the consequent current change in the Wheatstone bridge of the compensator. Similarly, the sensor tag gives a change of the modulating frequency of the backscattered signal in accordance with the real strain increment occurring in the gauge, but it depends on the reference frequency in unstressed condition, and it suffers from possible nonlinearity. In order to investigate these characteristics of the sensor tag, a large campaign of new tensile tests has been carried out making the initial frequency $f_{0}$ variable in a wide range of values. As explained in [30], this is possible operating a suitable tuning of the electrical resistance of the circuit. Tensile tests are extended to specimens made of steel, brass, and aluminium, searching for a general validation of the proposed method. As already done in Figure 7, the linear regressions are calculated on the experimental data and the angular coefficient $E_{\text {app }}=\Delta \sigma / \Delta f$ of each of these regressions is regarded as an apparent value of elastic modulus for the tested specimens. Results of these calculations, made with respect to a significant number of different $f_{0}$ values and extended to all the considered materials, have been reported in Figure 9. The ratio $k=E_{\text {app }} / E_{\text {ref }}$ among the calculated values of apparent elastic modulus $E_{\text {app }}$ and the reference value of the Young elastic modulus $E_{\text {ref }}$ (previously determined for various materials using the wired method) have been plotted with respect to the considered initial frequency $f_{0}$. In particular, a unique linear relationship has been highlighted among experimental $k$ values and $f_{0}$ ones, independently from the considered materials. That relationship can also be written as

$$
k=\frac{E_{\mathrm{app}}}{E_{\mathrm{ref}}}=\frac{\Delta \sigma / \Delta f}{\Delta \sigma / \Delta \varepsilon}=\frac{\Delta \varepsilon}{\Delta f}
$$

so that the variable $k$ represents the ratio among the real strain increment to be measured $(\Delta \varepsilon)$ and the measured 


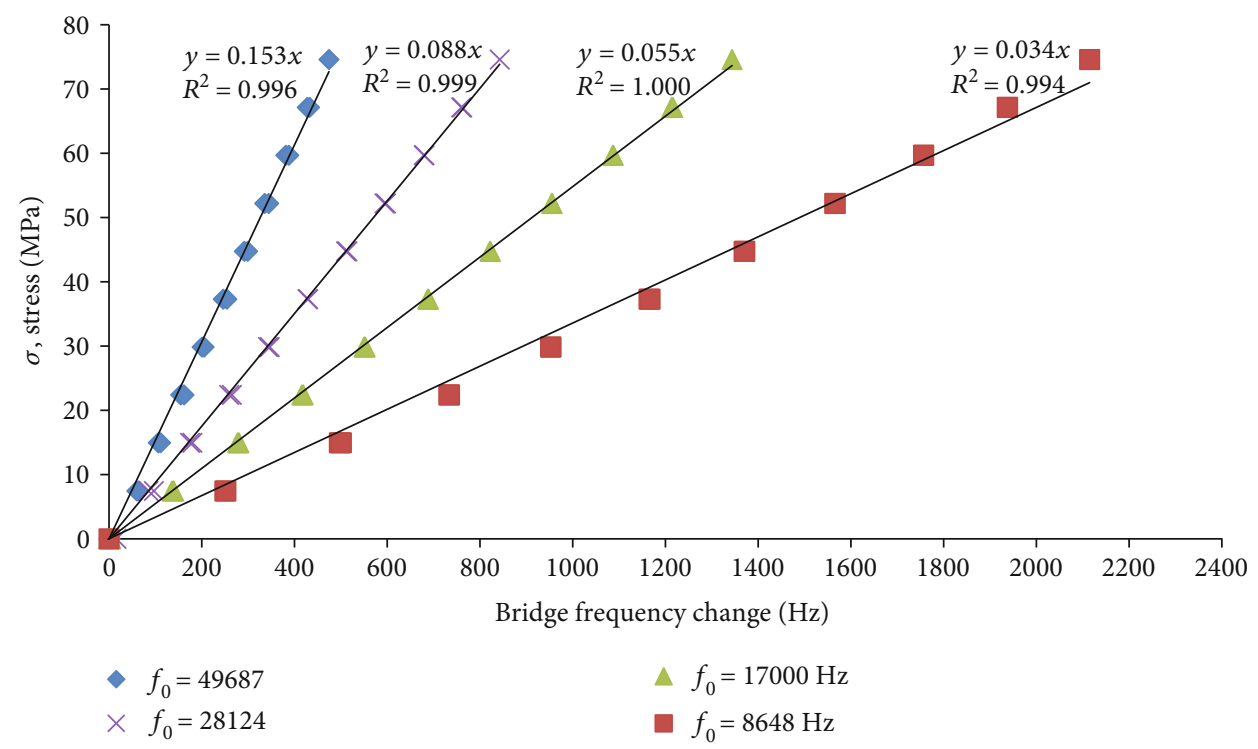

Figure 8: Effect of the tuning of the sensor tag. The frequency $f_{0}$ in unstressed condition depends on specific circuital parameters.

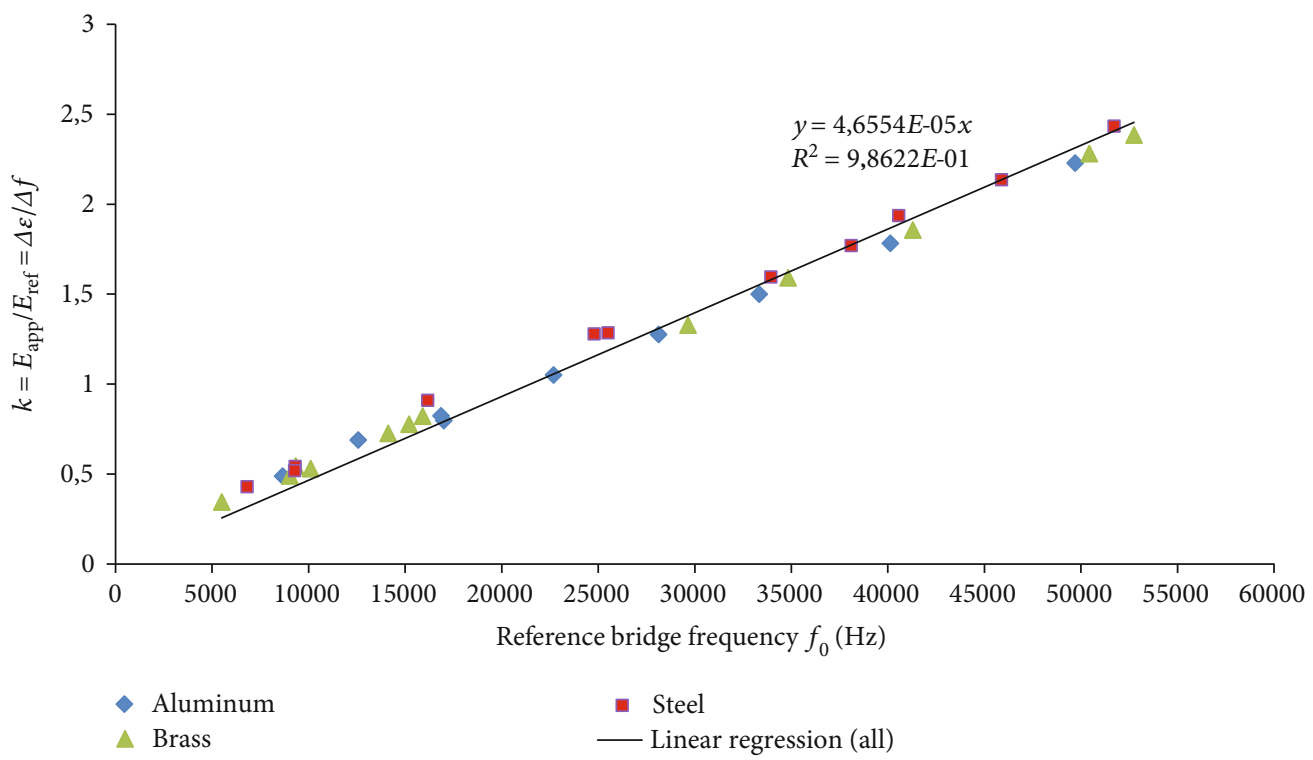

FIGURE 9: Experimental values for $k=E_{\text {app }} / E_{\text {ref }}$ as a function of the initial frequency $f_{0}$. Values of $k$ also express the ratio among the real strain increment in the gauge $(\Delta \varepsilon)$ and the measured frequency variation $\Delta f$.

frequency change $\Delta f$. This ratio depends on the considered frequency $f_{0}$. Then, linear regression calculated in Figure 9 provides the calibrating law to translate the measured frequency changes into the actual strain variation experienced by the specimen.

If nonlinearity of the experimental $\Delta \sigma-\Delta f$ curves is taken into account, at each step $i$ of a single tensile test, stress increments $\Delta \sigma_{i}=\sigma_{i+1}-\sigma_{i}$ and, consequently, single real strain increments $\Delta \varepsilon_{i}=\varepsilon_{i+1}-\varepsilon_{i}$ may be directly related to the correspondent frequency variations $\Delta f_{i}=f_{i+1}-f_{i}$. This allows to compute several local values of the apparent elastic modulus, here named secant values $E_{\text {sec }, i}=E_{\text {sec }}\left(f_{i}\right)=\left(\sigma_{i+1^{-}}\right.$ $\left.\sigma_{I}\right) /\left(f_{i+1}-f_{i}\right)$ which depends from the actual frequency $f_{i}$ at the step $i$. Consequently, a new definition for the ratio $k$ (Equation (2)) can be given with respect to the $E_{\mathrm{sec}, i}$ values in place of the $E_{\text {app }}$ ones, highlighting the continued dependence of ratio $k=E_{\mathrm{sec}} / E_{\text {ref }}$ from the current $f_{i}$ value in place of the initial value $f_{0}$. In these terms, ratio $k$ can be regarded as a continue function of the actual bridge frequency $f_{i}$ in accordance with the following expression:

$$
k=k\left(f_{i}\right)=\frac{E_{\mathrm{sec}}\left(f_{i}\right)}{E_{\mathrm{ref}}}=\frac{\left(\sigma_{i+1}-\sigma_{i}\right) /\left(f_{i+1}-f_{i}\right)}{\left(\sigma_{i+1}-\sigma_{i}\right) /\left(\varepsilon_{i+1}-\varepsilon_{i}\right)}=\frac{\Delta \varepsilon_{i}}{\Delta f_{i}} .
$$

Equation (3) highlights the existing relation between the real strain increment to be measured $\Delta \varepsilon_{i}$ and the frequency change $\Delta f_{i}$ measured in place of it. The ratio $k$ between 
these two variables is modelled as a continuous function of the instantaneous frequency $f$ while strain increments (and consequent frequency changes) are taking place.

A plot of $k=k\left(f_{i}\right)$ calculated for each tensile test is shown in Figure 10.

In particular, experimental $\Delta \sigma$ vs. $\Delta f$ curves recorded from tensile tests were first considered as sequences of individual steps $i$, whose stress increments $\Delta \sigma_{i}$ could be converted into the real strain variations $\Delta \varepsilon_{i}$ in accordance with Hooke's law $\Delta \varepsilon_{i}=\Delta \sigma_{i} / E_{\text {ref }}$ for each specific material. Subsequently, the values of the ratio $k=\Delta \varepsilon_{i} / \Delta f_{i}$ among the calculated real strain variations $\Delta \varepsilon_{i}$ and the related frequency changes $\Delta f_{i}$ recorded at the step $i$ have been computed and plotted with respect to the instantaneous bridge frequency value $f_{i}$ considered at the beginning of each considered step $i$.

Data in Figure 10 refer to the entire bunch of experimental values obtained from tensile tests performed on all the three different couples of metal specimen. These data clearly show the real strain increment and the correspondent frequency change to be strictly related to each other. In particular, several regression laws have been calculated on the experimental data, including an exponential form, a second order polynomial, and a linear equation, respectively. The first two regression laws showed to fit experimental data not significantly better than the linear regression one, this latter being characterized from an $R^{2}$ value very close to unity.

Assuming $k=k\left(f_{i}\right)$ as a continue function of $f_{i}$, the differential $k=\Delta \varepsilon / \Delta f$ can be considered and the real strain variation $\Delta \varepsilon$ is calculated in accordance with the following integral expression:

$$
\Delta \varepsilon=\int k(f) d f .
$$

If the linear regression law $k=2.382 E-05 \cdot f$ (indicated in Figure 10) is substituted in Equation (4), the strain increment $\Delta \varepsilon$ occurring in the gauge while the measured frequency changes from value $f_{1}$ to value $f_{2}$ is simply calculated as

$$
\Delta \varepsilon=1.1912 \cdot 10^{-5}\left(f_{2}^{2}-f_{1}^{2}\right) .
$$

Once again, it is worth noticing that Equation (5) is independent from the material of the specimen. Also, the validity of Equation (5) extends to a very wide range of frequency values (from just a few $\mathrm{Hz}$ up to $60 \mathrm{kHz}$ ). On the other hand, measurements performed with a sensor tag having lower initial frequency result to be more sensitive than that having higher initial frequency. In fact, in the former case, the ratio $k=\Delta \varepsilon / \Delta f$ is less than unity. In this condition, the frequency variation of $1 \mathrm{~Hz}$ corresponds to a strain variation lower than $1 \varepsilon$, representing a great improvement with respect to strain measurements performed with traditional techniques. In fact, electrical current variations in gauges are generally detected with such a limited resolution that only a precision of several $\varepsilon$ may correspond, this representing a limit for the traditional method of measuring strains with wired gauges. In comparison, the proposed new method may enhance the strain measurement resolution even below 0,2 $\varepsilon$ if the initial frequency is set lower than about $10 \mathrm{kHz}$.

4.4. Experimental Data from the Wireless Method: Dynamic Measurements. A steel cantilever about $25 \mathrm{~cm}$ long has been anchored at one end to the structure of a steel staircase as shown in Figure 11 so that it can vibrate after an initial displacement of its free end. The cantilever is instrumented with the proposed sensor that changes the modulation frequency of the backscattered signal dynamically according to the vibration movement. In fact, the strain gauge alternates extension and compression in agreement with the natural frequency of the beam while the backscattered signal changes its modulating frequency according to the extension and compression of the strain gauge. The analog modulated signal coming back from the sensor is received, sampled, and recorded by a Universal Software Radio Peripheral (National Instruments NI USRP 2920). A subsequent demodulation and filtering permit to determine the vibration frequency and the damping of the vibration. Figure 12 shows the modulation frequency of the received signal with respect to time in the case of repeated bending (with a pulse load) of the free end of the cantilever with a time interval of about 5 seconds. After each bending, the load is quickly removed permitting the cantilever to have free vibration. Figure 12 shows four responses of the cantilever under repeated loading and free vibration. Figure 13 shows an enlargement of the third response. Until about $13.8 \mathrm{~s}$ the cantilever is static, the small ripple concerns hardware and numerical noise. From $13.8 \mathrm{~s}$ until about $14.1 \mathrm{~s}$, the modulation frequency increases because of the progressive loading of the free end of the cantilever. After $14.1 \mathrm{~s}$, the load is quickly removed and the cantilever is under free vibration; the modulation frequency becomes oscillating since it follows the alternating extension and compression of the strain gauge glued on the cantilever. The frequency of that oscillation corresponds to the natural frequency of the cantilever as shown in Figure 14 that is the spectrum of the signal of Figure 13. The continuous component, in Figure 14, depends on the part of the signal corresponding to the static condition of the cantilever, the peak at $33.14 \mathrm{~Hz}$ is the natural frequency of vibration of the cantilever, and the small peak at $50 \mathrm{~Hz}$ is the hardware noise (from supplying network). Because of the damping of vibration, the oscillation of Figure 13 (i.e., the modulation frequency) decreases along the time. The response of the sensor has been compared to that measured by a laser detector (also shown in Figure 12) obtaining excellent agreement (shown in Figure 15).

\section{Conclusions}

A new method for measuring strains has been proposed. It consists in a wireless technique based on the traditional strain gauges and integrated into an electrical circuit together with an RFID tag. While traditionally strain measurement is usually performed by reading the current variation occurring in the circuit as a consequence of the resistance change in the strain gauge, in the proposed new method, an interrogating antenna is used to detect the modulation frequency of an 


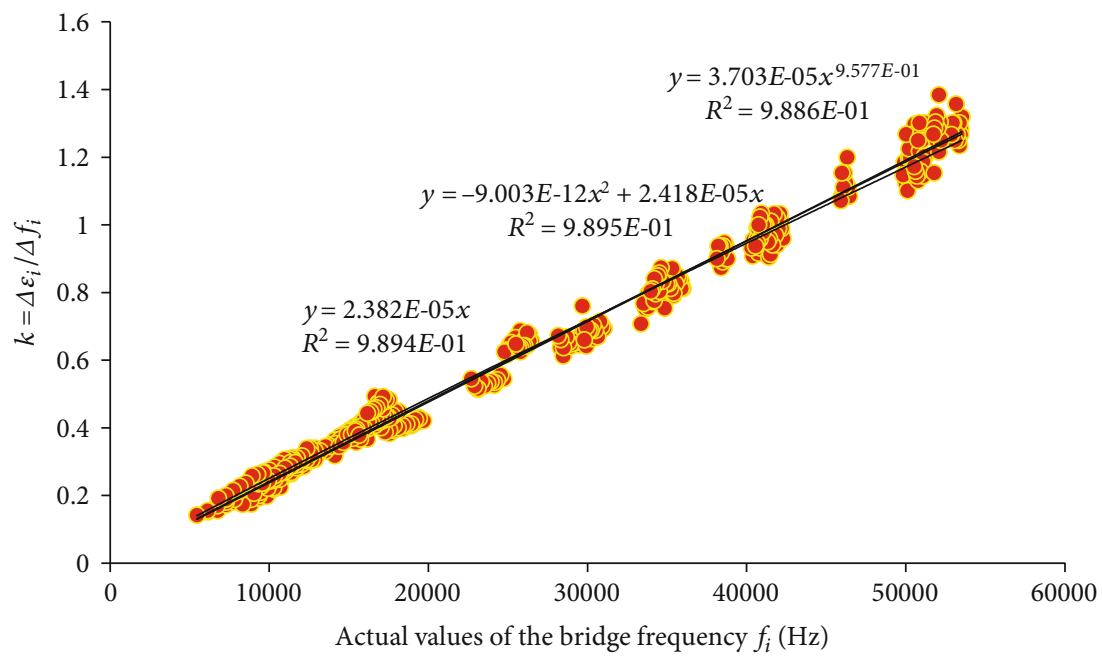

FIGURE 10: Different regressions carried out on experimental values of the ratio $k=\Delta \varepsilon_{i} / \Delta f_{i}$ considered as a function of the current bridge frequency $f_{i}$.

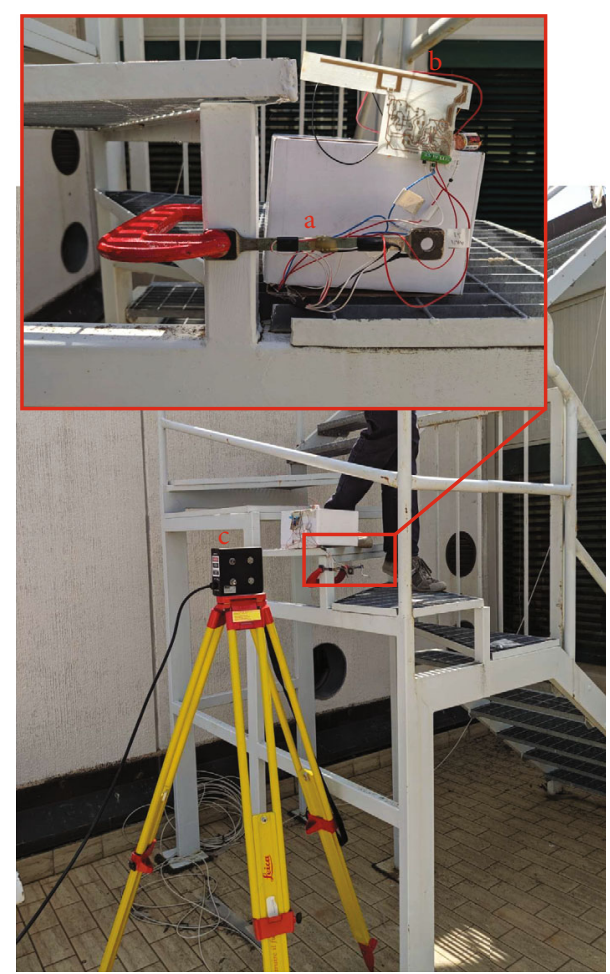

Figure 11: Dynamic measurement set-up: (a) instrumented cantilever anchored at one end to the structure of a steel staircase; (b) wireless sensor tag; (c) laser detector.

electromagnetic signal that varies in accordance with the strain to be measured.

Several tensile tests have been carried out on metal specimens made of different materials, including steel, brass, and aluminium. Feasibility of this new method has been proved determining a unique clear relationship among the strain values and the measured frequency changes.

The tag sensor is able to measure dynamic phenomena as assessed by experiments with vibrations of tens of $\mathrm{Hz}$. The

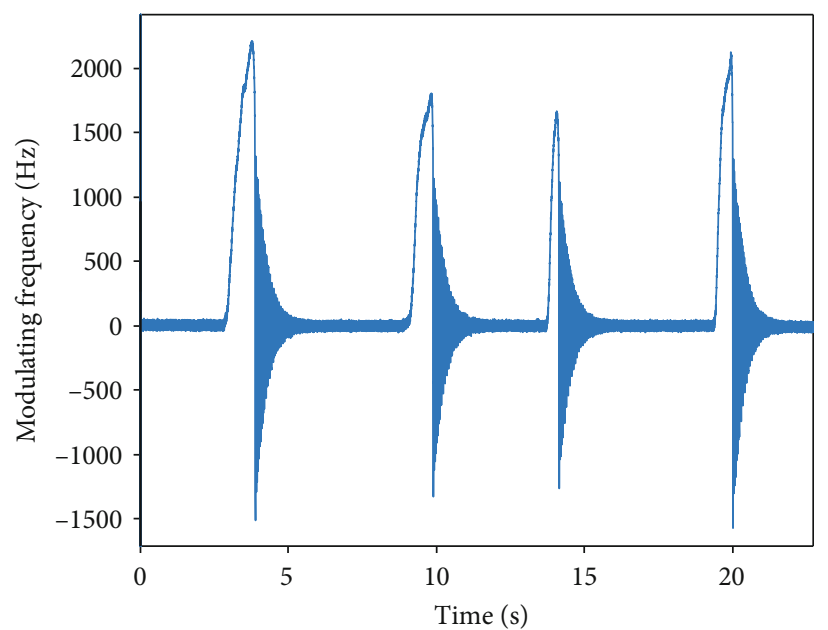

FIGURE 12: Response of the oscillating cantilever under repeated pulse loading. The enlargement of free vibration is shown in Figure 13.

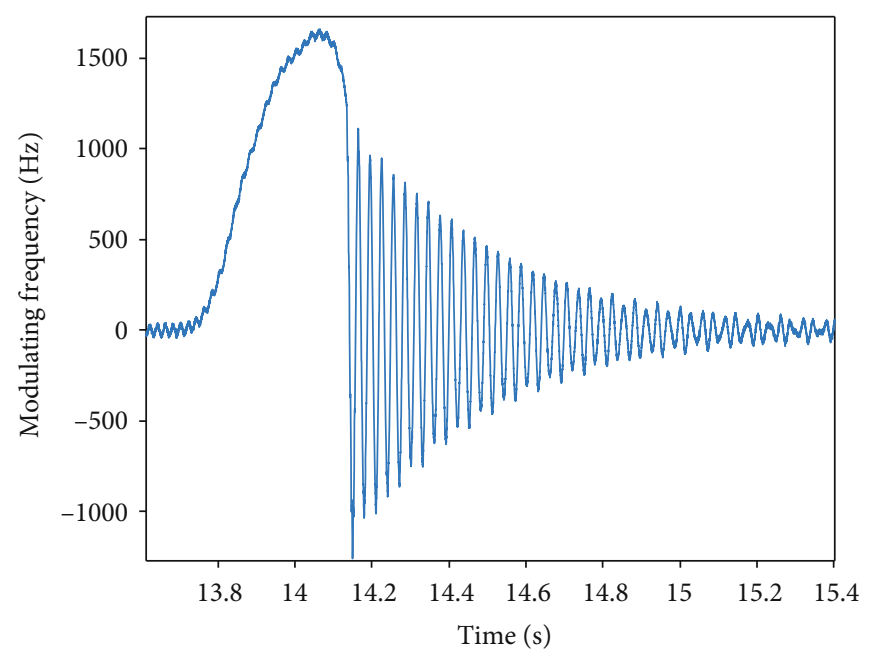

FIGURE 13: Enlargement of the third response of Figure 12. It shows the loading and free vibration of cantilever. 


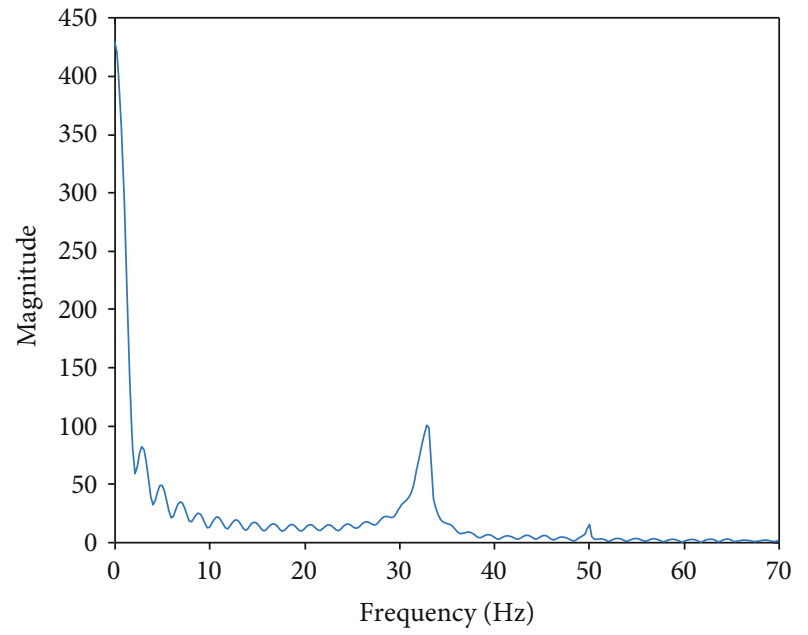

Figure 14: Spectrum of the signal of Figure 13: the continuous component (frequency zero) depends on the part of the signal corresponding to the static condition of the cantilever, the peak at $33.14 \mathrm{~Hz}$ is the natural frequency of vibration of cantilever, and the small peak at $50 \mathrm{~Hz}$ is the hardware noise (supplying network of the central unit).

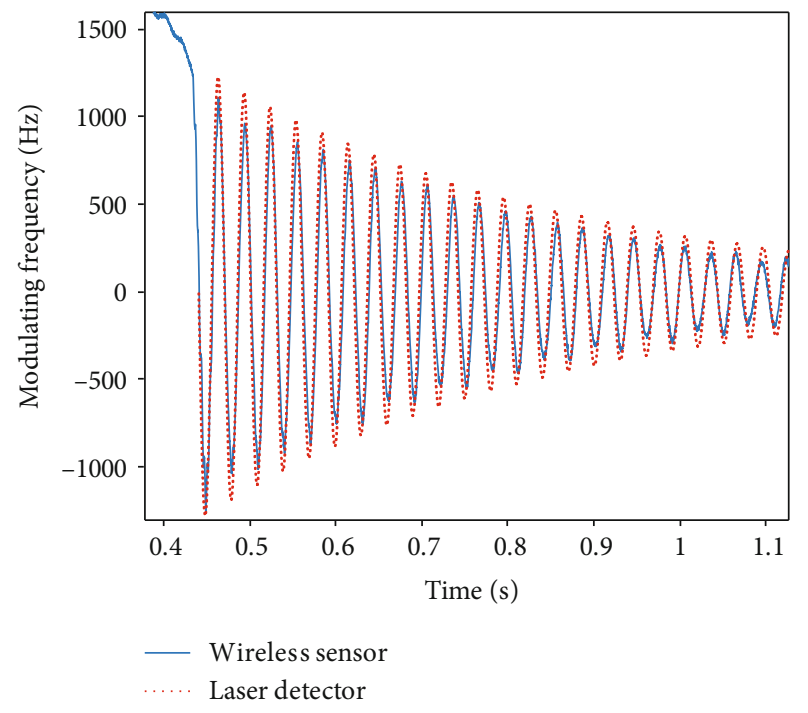

FIGURE 15: Comparison between the response of the wireless sensor and measurement with a laser detector (after a suitable scaling and translation).

detectable frequency of vibration is not limited by the sampling rate because the sampling is performed in the central unit (not in the tag sensor) that can be equipped with a suitable sampling device. The limit in measuring oscillating phenomena is given by the bandwidth of the strain gauge that is in the order of $\mathrm{KHz}$, while the bandwidth of electronic components is much larger in the order of MHz. So, the tag sensor should be able to measure oscillations up to a few $\mathrm{kHz}$.

A calibration of the measuring system has been proposed, showing that it remains valid for a large range of working frequencies and for large strain intervals as well. In comparison with a traditional strain measuring procedure, the accuracy of the proposed new technique has been proved to be potentially higher. Effectiveness of the proposed wireless method has been proved up to a maximum interrogating distance of 20 meters in a laboratory room and outdoor, making this new strain measuring technique suitable for structural monitoring.

\section{Data Availability}

The data used to support the findings of this study are included within the article.

\section{Conflicts of Interest}

The authors declare that there is no conflict of interest regarding the publication of this paper.

\section{Acknowledgments}

This article was supported by the Department of Civil, Building and Environmental Engineering of the Università degli Studi dell'Aquila and the Department of Industrial and Information Engineering and Economics of the University of L’Aquila, Via Giovanni Gronchi 18, 67100, L'Aquila, Italy.

\section{References}

[1] B. F. Spencer, "Opportunities and challenges for smart sensing technology," in Proceedings of the First International Conference on Structural Health Monitoring and Intelligent Infrastructure, pp. 65-71, Japan. A A Balkema Publisher, 2003.

[2] E. G. Straser, A. S. Kiremidjian, T. H. Meng, and L. Redlefsen, "A modular, wireless network platform for monitoring structures," in SPIE Vol. 3243, Proceedings of the 16th International Modal Analysis Conference, pp. 450-456, 1998.

[3] J. P. Lynch, A. Sundararajan, K. H. Law, A. S. Kiremidjian, T. Kenny, and E. Carryer, "Embedment of structural monitoring algorithms in a wireless sensing unit," Structural Engineering and Mechanics, vol. 15, no. 3, pp. 285-297, 2003.

[4] K. Mitchell, S. Sana, V. S. Balakrishnan, V. S. Rao, and H. J. Pottinger, "Micro sensors for health monitoring of smart structures," in Proceedings Volume 3673, Smart Structures and Materials 1999: Smart Electronics and MEMS, pp. 351358, Newport Beach, CA, USA, July 1999.

[5] Y. Yu, H. W. Li, and J. P. Ou, "Wireless acceleration sensor used for civil engineering structure monitoring and its integration technique," Proceedings of the 3rd International Symposium on Instrumentation Science and Technology, 2004, pp. 741-748, Harbin Institute of Technology Publisher, 1, China, 2004.

[6] Y. Yu, H. W. Li, and J. P. Ou, "Design and development of wireless acceleration sensor applied to civil engineering structure," Journal of Transcluction Technology, 2004.

[7] G. Park, T. Rosing, M. D. Todd, C. R. Farrar, and W. Hodgkiss, "Energy harvesting for structural health monitoring sensor networks," Journal of Infrastructure Systems, vol. 14, no. 1, pp. 64-79, 2008.

[8] M. Jeong, J.-G. Bae, and B.-H. Koh, "A feasibility study of damage tracking through the diffusive communication of wireless sensors," International Journal of Precision Engineering and Manufacturing, vol. 11, no. 1, pp. 23-29, 2010. 
[9] L. Liu and F. G. Yuan, "Wireless sensors with dual-controller architecture for active diagnosis in structural health monitoring," Smart Materials and Structures, vol. 17, no. 2, article 025016, 2008.

[10] R. Melik, E. Unal, N. Kosku Perkgoz, C. Puttlitz, and H. V. Demir, "Flexible metamaterials for wireless strain sensing," Applied Physics Letters, vol. 95, no. 18, article 181105, 2009.

[11] R. Melik, N. K. Perkgoz, E. Unal, C. Puttlitz, and H. V. Demir, "Bio-implantable passive on-chip RF-MEMS strain sensing resonators for orthopaedic applications," Journal of Micromechanics and Microengineering, vol. 18, no. 11, article 115017, 2008.

[12] I. Mohammad and H. Huang, "An antenna sensor for crack detection and monitoring," Advances in Structural Engineering, vol. 14, no. 1, pp. 47-53, 2011.

[13] J.-T. Lin, K. W. Walsh, D. Jackson et al., "Development of capacitive pure bending strain sensor for wireless spinal fusion monitoring," Sensors and Actuators A, vol. 138, no. 2, pp. 276287, 2007.

[14] F. Gasco, P. Feraboli, J. Braun, J. Smith, P. Stickler, and L. DeOto, "Wireless strain measurement for structural testing and health monitoring of carbon fiber composites," Composites Part A: Applied Science and Manufacturing, vol. 42, no. 9, pp. 1263-1274, 2011.

[15] P. C. Chang, A. Flatau, and S. C. Liu, "Review paper: health monitoring of civil infrastructure," Structural Health Monitoring, vol. 2, no. 3, pp. 257-267, 2003.

[16] A. Kesavan, S. John, and I. Herszberg, "Strain-based structural health monitoring of complex composite structures," Structural Health Monitoring, vol. 7, no. 3, pp. 203-213, 2008.

[17] U. Tata, S. Deshmukh, J. C. Chiao, R. Carter, and H. Huang, "Bio-inspired sensor skins for structural health monitoring," Smart Materials and Structures, vol. 18, no. 10, article 104026, 2009.

[18] A. Ibrahim and D. R. S. Cumming, "Passive single chip wireless microwave pressure sensor," Sensors and Actuators A: Physical, vol. 165, no. 2, pp. 200-206, 2011.

[19] R. Matsuzaki, M. Melnykowycz, and A. Todoroki, "Antenna/sensor multifunctional composites for the wireless detection of damage," Composites Science and Technology, vol. 69, no. 1516 , pp. 2507-2513, 2009.

[20] Y.-s. H. Son and J.-G. Kim, "A design and implementation of forest-fires surveillance system based on wireless sensor networks for South Korea Mountains," IJCSNS International Journal of Computer Science and Network Security, vol. 6, no. 9B, 2006.

[21] O. Jinping, "Research and practice of intelligent sensing technologies in civil structural health monitoring in the mainland of China," in Proceedings Volume 6176, Nondestructive Evaluation and Health Monitoring of Aerospace Materials, Composites, and Civil Infrastructure V, San Diego, CA, USA, March 2006.

[22] Y. Yu and J. Ou, "Wireless sensing experiments for structural vibration monitoring of offshore platform," Frontiers of Electrical and Electronic Engineering in China, vol. 3, no. 3, pp. 333-337, 2008.

[23] S. J. Dyke, J. M. Caicedo, and E. A. Johnson, "Monitoring of a benchmark structure for damage identification," in Proceedings of the Engineering Mechanics Speciality Conference, pp. 21-24, Austin, TX, USA, May 2000.

[24] Stanford University, USA, "Wireless structural damage monitoring," Smart Materials Bulletin, vol. 2002, no. 1, p. 16, 2002.
[25] J. P. Lynch and K. Loh, "A summary review of wireless sensors and sensor networks for structural health monitoring," The Shock and Vibration Digest, vol. 38, no. 2, pp. 91-128, 2006.

[26] D. D. L. Mascarenas, E. B. Flynn, M. D. Todd et al., "Development of capacitance-based and impedance-based wireless sensors and sensor nodes for structural health monitoring applications," Journal of Sound and Vibration, vol. 329, no. 12, pp. 2410-2420, 2010.

[27] E. Köppe and M. Bartholmai, "Wireless sensor network with temperature compensated measuring technology for longterm structural health monitoring of buildings and infrastructures," Procedia Engineering, vol. 25, pp. 1032-1036, 2011.

[28] S.-D. Jang and J. Kim, "Passive wireless structural health monitoring sensor made with a flexible planar dipole antenna," Smart Materials and Structures, vol. 21, no. 2, article 027001, 2012.

[29] S.-D. Jang, B.-W. Kang, and J. Kim, "Frequency selective surface based passive wireless sensor for structural health monitoring," Smart Materials and Structures, vol. 22, article 025002, no. 2, 2013.

[30] E. DiGiampaolo, A. DiCarlofelice, and A. Gregori, “An RFIDenabled wireless strain gauge sensor for static and dynamic structural monitoring," IEEE Sensors Journal, vol. 17, no. 2, pp. 286-294, 2017. 


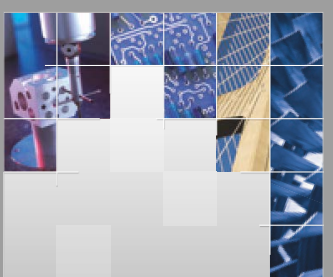

\section{Enfincering}
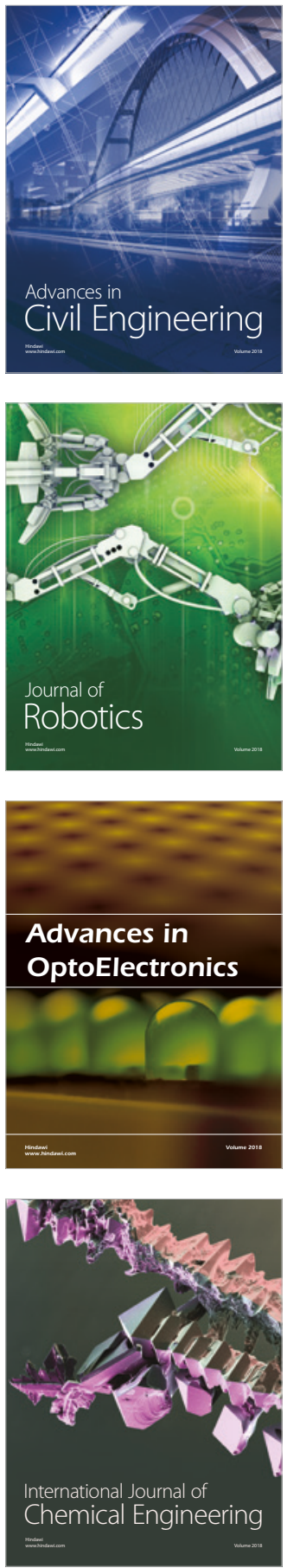

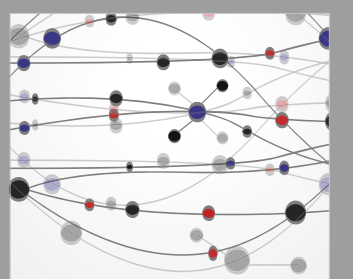

\section{Rotating \\ Machinery}

The Scientific World Journal

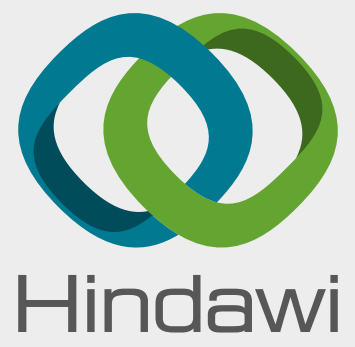

Submit your manuscripts at

www.hindawi.com
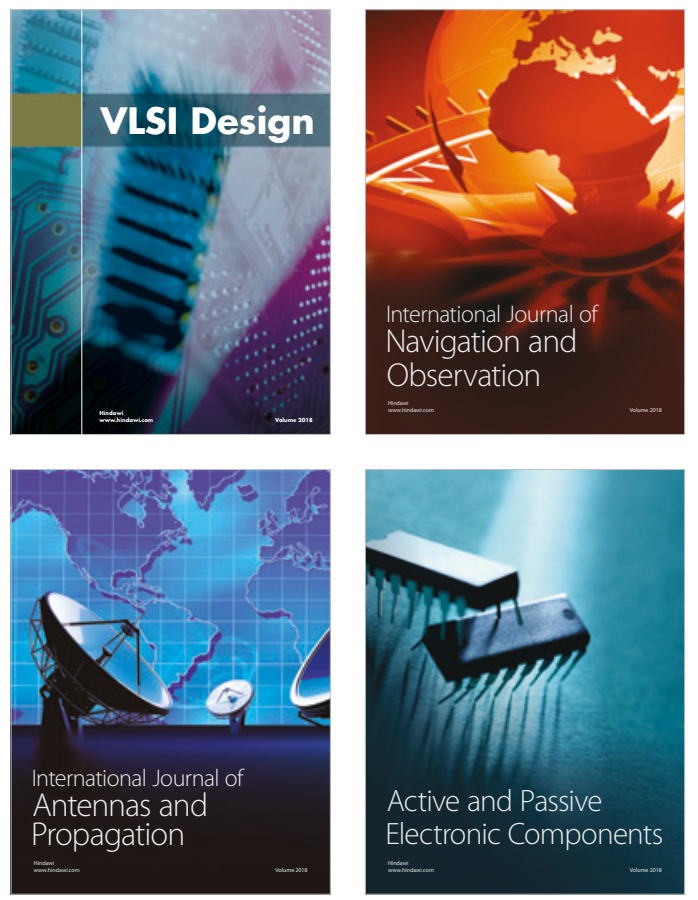
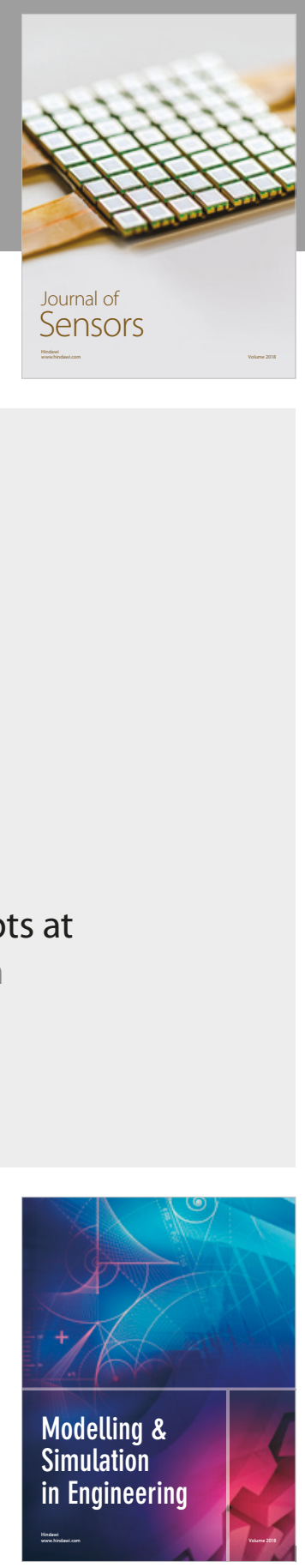

\section{Advances \\ Multimedia}
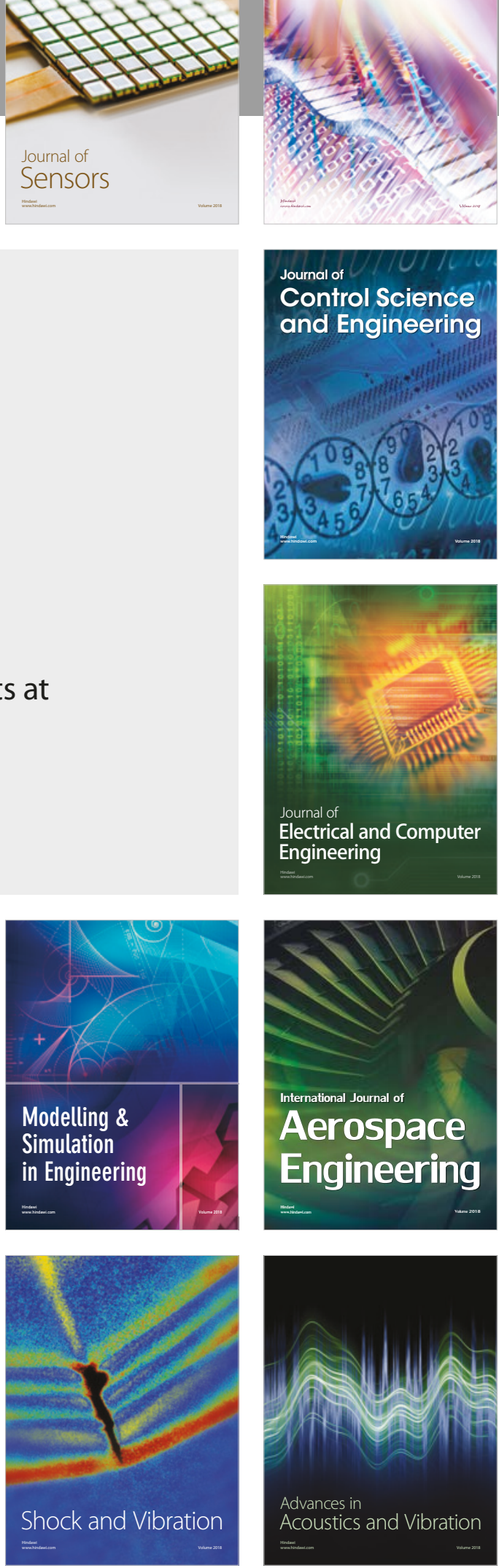